\title{
Bateria Psicológica para Avaliação da Atenção (BPA): desempenho de diferentes faixas etárias
}

\author{
Fabián Javier Marín Rueda - Universidade São Francisco, Itatiba, Brasil \\ Rebecca de Magalhães Monteiro - Faculdade Pitágoras, Belo Horizonte, Brasil
}

\begin{abstract}
Resumo
O objetivo do estudo foi analisar o desempenho atencional em diferentes momentos da vida. Participaram 1.759 pessoas, de ambos os sexos, e com idades variando de 6 até 82 anos. O instrumento utilizado foi a Bateria Psicológica para Avaliação da Atenção (BPA), composta pelos testes de Atenção Concentrada, Atenção Dividida e Atenção Alternada. Os resultados estão de acordo com o apontado pela literatura ao relacionar a atenção com as diferentes etapas do desenvolvimento. Nesse sentido, observou-se que houve um aumento de desempenho até o início da vida adulta, com um leve decréscimo a partir dos 30 anos, que fica mais evidente a partir dos 50 anos. Ainda, a ANOVA permitiu identificar seis faixas etárias que se diferenciaram claramente. Por fim, identificou-se que o desempenho em atenção dividida é inferior aos outros tipos de atenção. Sugerem-se novos estudos que permitam identificar e diferenciar um maior número de idades.
\end{abstract}

Palavras-chave: Atenção, Desenvolvimento, Validade, Testes psicológicos.

Psychological Battery for Attention Assessment (BPA): performance of different age groups

\begin{abstract}
This study aimed to analyze the attentional performance at different periods of life. The participants consisted of 1.759 people, of both sexes, and ages ranging from 6 to 82 years old. The instrument used was the Psychological Battery for Attention Assessment (BPA), composed of the tests of Concentrated Attention, Divided Attention and Alternate Attention. The results are in agreement with that was indicated by the literature, relating attention to the different stages of development. In that sense, it was observed there was a performance increase up to early adulthood, a slight decrease from the $30 \mathrm{~s}$, which becomes more evident after 50s. Still, the ANOVA identified six age groups that clearly differed. Finally, we found that the performance in divided attention is lower than for other types of attention. We suggest further studies to identify and differentiate a large number of ages.

Keywords: Attention; Development; Validity; Psychological tests.
\end{abstract}

Batería Psicológica para Evaluación de la Atención (BPA): desempeño de distintos rangos etarios

\begin{abstract}
Resumen
El objetivo del estudio fue analizar la capacidad de atención en diferentes momentos de la vida. Participaron 1.759 personas, de ambos sexos, y con edades de 6 hasta 82 años. El instrumento utilizado fue la Batería Psicológica para Evaluación de la Atención (BPA), compuesta por los testes de Atención Concentrada, Atención Dividida y Atención Alternada. Los resultados están de acuerdo con lo mencionado por la literatura al relacionar la atención con las distintas etapas del desarrollo. En ese sentido, se observó que hubo un aumento en el desempeño hasta el inicio de la vida adulta, con una pequeña disminución a partir de los 30 años, que se queda más evidente a partir de los 50 años. Además, el ANOVA permitió identificar seis rangos etarios que se diferencian claramente. Finalmente, se identificó que el desempeño en atención dividida es inferior a los otros tipos de atención. Se sugieren nuevos estudios que permitan identificar y diferenciar un mayor número de edades.

Palabras-clave: Atención, Desarrollo, Validez, Testes psicológicos.
\end{abstract}

Nas últimas décadas, várias pesquisas em Psicologia têm produzido informações sobre diferentes fenômenos relacionados ao construto atenção (Allport, 1993; Davies \& Parasuraman, 1982; Eysenck \& Keane, 2007; Fu, Fedota, Greenwood \& Parasuraman, 2010; Pashler, 1999; Posner, 1993; Richards, 2005; Sternberg, 2008; Treisman \& Gelade, 1980; Warm, 1984; entre outros). Dentre os fenômenos investigados encontramse as diferentes taxonomias adotadas por pesquisadores da área, no intuito de explicar a complexidade e a operacionalização do construto.

Sob essa perspectiva, a atenção pode ser classificada de acordo com a capacidade do sujeito de centrar-se em um estímulo importante, suprimindo de forma deliberada estímulos distratores em um tempo predeterminado (Baños \& Belloch, 1995; Zillmer \& Spiers, 1998), a capacidade em distribuir a atenção entre diferentes estímulos ao mesmo tempo (Dalgalarrondo, 2000; Sternberg, 2008; Wagner, 2003), bem como a capacidade de ora manter o foco de atenção em um estímulo ora em outro (Dalgalarrondo, 2000; Wagner, 2003). De acordo com os autores citados, essas classificações são denominadas de Atenção Concentrada, Atenção Dividida e Atenção Alternada, respectivamente.

Vale salientar que as definições que fundamentam a operacionalização de cada tipo de atenção sofrem algumas variações, de acordo com a teoria que as postula. A exemplo disso, Lezak (1995) afirma que um teste como o D2, que se propõe avaliar a atenção concentrada, na Alemanha é conhecido como 'Teste de Concentração' ou 'Teste de Atenção Seletiva', enquanto nos Estados Unidos recebe o nome de 'Teste de Amplitude Atencional', 'Teste de Atenção Seletiva' 
ou 'Teste de Atenção Sustentada'. Também, autores como Zillmer e Spiers (1998) apontam que muitas vezes a atenção seletiva é definida como atenção concentrada. Sendo assim, ressalta-se que, como em vários outros construtos da ciência psicológica, embora as teorias e definições sobre a atenção sejam amplas, não há um consenso entre os pesquisadores em relação à melhor definição para o construto.

Outro fenômeno relevante estudado por pesquisadores da área é a influência da variável idade no desempenho em testes de atenção. Para Richards (2005), a atenção pode ser considerada uma função cognitiva que ocorre desde os primeiros dias de vida, sendo sua principal função orientar os sentidos aos estímulos do ambiente. Dessa maneira, na medida em que o cérebro se desenvolve, passa a administrar de forma seletiva os recursos de processamento da informação, isto é, prestar atenção em um estímulo e inibir outros. Ao lado disso, vale destacar que segundo Papalia e Olds (2000) e Sanchez-Gil e Perez-Martinez (2008), pesquisas relacionadas ao desenvolvimento cognitivo sugerem acréscimos, em termos de desempenho, desde a infância até a idade de adulto jovem, seguidos de perdas significativas decorrentes do processo de envelhecimento, que se acentuam na fase que compreende a terceira idade. Nesse sentido, é possível dizer que a capacidade atentiva sofre alterações significativas nas diferentes fases da vida.

Ao investigarem a relação entre idade e processo atencional, por meio de duas tarefas de atenção (dividida e alternada), ambas contendo medidas visuais e auditivas apresentadas separadamente, Hawkins, Kramer e Capaldi (1992) encontraram diferenças significativas de acordo com a faixa etária. Participaram da pesquisa dois grupos com 14 sujeitos cada, um entre 20 e 35 anos, e outro entre 67 e 74. Quando comparado o tempo de reação dos grupos, os autores observaram que os adultos jovens foram sistematicamente mais rápidos que os idosos.

O estudo de Pesce, Guidetti, Baldari, Tessitore e Capranica (2005) teve como objetivo investigar a relação entre idade e atenção. Para tanto, a pesquisa contou com a participação de três grupos com 14 sujeitos cada, cujas idades variaram de 12 a 15 anos, de 24 a 38 anos e de 60 a 75 anos, respectivamente. A tarefa consistiu em localizar um alvo previamente definido após uma dica visual. Em seus resultados, os autores verificaram que os idosos apresentaram, sistematicamente, um desempenho pior do que os adultos jovens e os adolescentes, mas não houve diferenças entre esses dois últimos grupos. Nesse sentido, os autores sugeriram que o pior desempenho verificado nos idosos poderia ser resultado de deficiências orgânicas decorrentes do envelhecimento.
Em uma pesquisa com o teste de Atenção Sustentada, Rueda, Noronha, Sisto e Bartholomeu (2008) buscaram evidência de validade por meio da verificação da performance ao longo de diferentes idades para as medidas de concentração e de velocidade com qualidade. Foram participantes 127 candidatos indivíduos com idades entre 20 e 47 anos, de diferentes cidades do Estado de Minas Gerais. Os resultados revelaram uma correlação negativa e significativa entre as idades e as medidas de concentração e velocidade com qualidade, respectivamente. Isso significa que, conforme o aumento da idade, houve uma diminuição de desempenho nas medidas investigadas, o que evidenciou uma relação entre a idade e essas medidas.

O estudo de Lima, Travaini e Ciasca (2009) teve como objetivo obter uma amostra referencial do desempenho de crianças sem dificuldades de aprendizagem, de ambos os sexos e faixa etária entre 7 e 10 anos de idade, em testes que avaliam a atenção e as funções executivas. Os instrumentos utilizados para avaliar a atenção foram o Teste de Cancelamento e Trail Making Test - Parte A. Para avaliar as funções executivas, os testes usados foram o Trail Making TestParte B, Stroop Color Word Test e Torre de Londres. Por fim, para as habilidades escolares foi selecionado o Teste de Desempenho Escolar. Os resultados revelaram efeitos da idade e da série escolar no desempenho dos testes, principalmente nos escores de tempo, uma vez que com o avanço da faixa etária e nível de escolaridade, o desempenho melhorou significativamente.

$\mathrm{Na}$ pesquisa de Rueda (2011), o objetivo foi verificar evidência de validade em função da variável idade para o Teste de Atenção Dividida, partindo da hipótese de que ao aumento da idade corresponderia uma diminuição da capacidade de dividir a atenção. Foram participantes do estudo 878 indivíduos dos Estados da Bahia e Sergipe, com idades entre 18 e 72 anos $(M=26,74, D P=8,14)$. Os resultados revelaram correlação negativa, significativa e de magnitude moderada $(r=-0,37 ; p<0,001)$ entre a pontuação do Teste de Atenção Dividida e a idade das pessoas. A análise de variância mostrou diferenças significativas entre as idades, permitindo agrupar três faixas etárias, diferenciadas pela prova de Tukey. As faixas etárias foram até 25 anos, de 26 a 35 anos e de 36 anos ou mais, e o desempenho diminuiu conforme o aumento da idade, comprovando assim a hipótese do autor.

Com o propósito de buscar dados normativos para o Teste de Atenção por Cancelamento (TAC), Hazin e cols., (2012) realizaram um estudo com 524 sujeitos, com idades entre sete e 16 anos, do ensino fundamental das redes pública e privada da cidade de Natal/RN. Foi observado que as variáveis nível de 
escolaridade, tipo de escola e faixa etária mostraram-se estatisticamente significativas, enquanto a variável sexo não apresentou relevância para diferenciação de desempenho dos estudantes. Em relação à acurácia e velocidade em tarefas que avaliam a seletividade e alternância, os resultados revelaram aumento significativo no desempenho dos estudantes nas três primeiras séries do ensino fundamental e estabilização dos resultados nos três últimos anos, sugerindo o estabelecimento de platô no desenvolvimento atencional. Com base nesses resultados, os autores concluíram que em razão da faixa etária da criança e do adolescente, do tipo de escola e nível de escolaridade, o instrumento em questão apresenta relevância para a avaliação dos sistemas atencionais, mostrando-se sensível para a detecção de aspectos neurodesenvolvimentais e características socioculturais do funcionamento cognitivo humano.

Ao considerar os resultados obtidos nas pesquisas relatadas, discute-se que um teste de avaliação da atenção desenvolvido para diferentes fases do desenvolvimento deve ser sensível para identificar as mudanças desenvolvimentais características do processo maturacional do cérebro infantil e, posteriormente, de deficiências orgânicas decorrentes do envelhecimento. Por outro lado, se esse mesmo instrumento pudesse considerar diferentes formas de operacionalização da atenção, tais como atenção concentrada, dividida e alternada, os dados decorrentes do processo avaliativo poderiam fornecer informações ainda mais aprofundadas sobre o fenômeno em questão. Assim sendo, e ao refletir sobre tais questões, foi objetivo deste estudo analisar o desempenho atencional em diferentes fases da vida por meio de uma bateria de testes de atenção, cuja operacionalização engloba três tipos, a saber, a concentrada, a dividida e a alternada.

\section{Método}

\section{Participantes}

Participaram do estudo 1.759 indivíduos de ambos os sexos, sendo 52,8\% do sexo feminino, dos estados da Bahia, Minas Gerais, São Paulo e Sergipe. No que se refere à escolaridade, ela abrangeu a Educação de Jovens e Adultos - EJA (11,2\%), o Ensino Fundamental completo e incompleto $(21,5 \%)$, o Ensino Médio completo ou incompleto $(21,2 \%)$, o Ensino Superior completo ou incompleto (43,1\%), assim como a Pós-Graduação. Em relação à idade, ela variou de 6 a 82 anos, com uma média de 29,61 e um desvio padrão de 16,43 . A distribuição e frequência das idades pode ser visualizada na Figura 1.

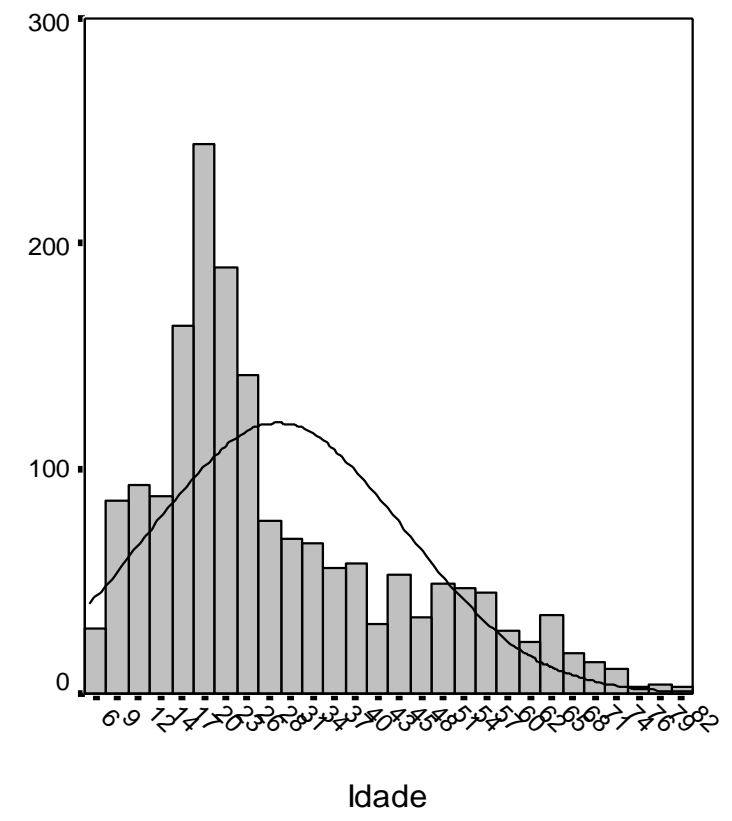

Figura 1. Distribuição dos participantes por idade

Pela Figura 1 pode ser observado que a maior parte dos indivíduos ficou concentrada entre 14 e 26 anos $(44,8 \%)$, observando-se que a partir dos seis anos houve um aumento de participantes em todas as idades até chegar aos 18 anos, idade na qual ocorreu a maior frequência de sujeitos $(6,2 \%)$. A partir dos 18 anos constata-se um decréscimo gradativo no número de sujeitos em cada idade, que fica mais evidente a partir dos 30 anos. Os participantes acima de 60 anos foram 124 , representando $6 \%$ da amostra. De forma geral, 
percebe-se que, embora exista uma diferença clara no número de participantes das diferentes idades, houve uma boa representação das idades dos participantes.

\section{Instrumentos}

Bateria Psicológica para Avaliação da Atenção - BPA (Rueda, 2013)

A Bateria Psicológica para Avaliação da Atenção (BPA) tem como objetivo realizar uma avaliação da capacidade geral de atenção, assim como uma avaliação individualizada de tipos de atenção específicos, quais sejam, atenção concentrada (AC), atenção dividida (AD) e atenção alternada (AA). Ela é composta por três testes, cada um deles tendo como objetivo avaliar um dos tipos atencionais propostos. Por sua vez, a análise dos três testes em conjunto fornece a medida de atenção geral.

Os testes foram construídos a partir de vários estímulos abstratos que serviram para compor as três folhas de respostas (AC, AD e AA), sendo que ora eles foram estímulos-alvo, ora estímulos distratores. A distribuição dos estímulos segue a mesma quantidade em cada instrumento, contendo 400 estímulos distribuídos em 20 linhas com 20 estímulos cada. Do total, 120 são estímulos-alvo (pontuação máxima possível) e 280 distratores.

$O$ resultado final de cada teste é obtido considerando os estímulos-alvo que a pessoa marcou, subtraído dos erros e das omissões que cometeu. Dessa forma chega-se ao total de pontos. No caso da capacidade geral da atenção, o resultado é obtido por meio da somatória dos pontos totais de cada um dos testes $(\mathrm{AC}+\mathrm{AD}+\mathrm{AA})$, sendo o máximo possível 360 pontos.

No que se refere ao tempo de aplicação, no caso do AC é 2 minutos; para o AD, 4 minutos; e para o AA, 2 minutos e 30 segundos. A ordem de aplicação deve ser seguida rigorosamente, começando pelo AC, seguido pelo $\mathrm{AD}$ e, por fim, o AA. A aplicação pode ser individual e coletiva.

No que se refere às propriedades psicométricas, os estudos de evidências de validade foram realizados comparando à BPA com o Teste de Atenção Concentrada (TEACO-FF), Testes de Atenção Dividida (TEADI) e Alternada (TEALT), Teste NãoVerbal de Inteligência - R-1 e Teste dos Cubos: para avaliação do raciocínio visuoespacial. Por sua vez, os índices de precisão foram obtidos pelo método de teste-reteste, com intervalo de uma e duas semanas.

Em relação ao estudo com os testes TEACO-FF, TEADI e TEALT, foi verificada evidência de validade convergente, uma vez que os coeficientes de correlação entre os testes que avaliavam o mesmo tipo de atenção foram todos superiores a 0,51 , valor esse considerado por Nunes e Primi (2010) como indicativo desse tipo de evidência. Por sua vez, ao correlacionar testes que avaliavam tipos diferentes de atenção, os coeficientes variaram entre 0,34 e 0,59 , demonstrando a obtenção de evidência de validade pela comparação entre testes que avaliam construtos relacionados, o que também foi ao encontro do apontado pela literatura.

Já no estudo realizado com o R-1, os coeficientes de correlação foram todos positivos, variando de 0,31 a 0,45 . Por sua vez, no estudo com o Teste dos Cubos os coeficientes também foram positivos e significativos, variando de 0,20 a 0,29, excetuando apenas a correlação com o AC da BPA, que apresentou um coeficiente nulo e não-significativo. Com base nisso, conclui-se pela obtenção de evidência de validade pela comparação com testes que avaliam construtos relacionados.

Por fim, a precisão pelo método de teste-reteste foi analisada para cada instrumento (AC, AD, AA e a Atenção Geral) e em três momentos, quais sejam, após sete dias da primeira aplicação, quatorze dias após a primeira aplicação, e comparando a segunda aplicação (7 dias) com a terceira (14 dias). Os coeficientes foram considerados bastante satisfatórios, variando de 0,68 a 0,89 . Ao considerar cada medida de forma separada, no caso do $\mathrm{AC}$ os valores variaram entre 0,69 e 0,85 ; no $\mathrm{AD}$ houve uma variação entre 0,66 e 0,89 ; entre 0,69 e 0,84 ficaram os coeficientes para o AA e, por fim, no caso da atenção geral, os valores do coeficiente de correlação de teste-reteste variaram entre 0,68 e 0,83. Dessa forma, os resultados de precisão obtidos na BPA foram satisfatórios e atestam a confiabilidade do instrumento.

\section{Procedimentos}

Antes de iniciar a investigação, o projeto de pesquisa foi submetido e aprovado por um Comitê de Ética, sob o número CAAE: 0189.0.142.000-09. Após a aprovação, os dados foram coletados em escolas públicas e particulares de ensino fundamental e médio, na EJA, em diferentes cursos superiores de universidades públicas e particulares, assim como em clínicas de avaliação psicológica para motoristas. No caso dessas clínicas, a aplicação dos instrumentos ocorreu de forma individual e durou em torno de 20 minutos. Já no restante das aplicações, as mesmas foram realizadas coletivamente, tiveram uma variação entre 25 e 50 minutos, dependendo da idade e do grau de escolaridade dos sujeitos.

\section{Resultados}


$\mathrm{Na}$ análise dos resultados, primeiramente foram realizados gráficos de dispersão para visualizar qual era relação existente entre as medidas de atenção e a variável idade. Posteriormente, foi conduzido um estudo para verificar quais as faixas etárias que melhor se diferenciavam, utilizando a prova de Tukey para verificar a pontuação das faixas que explicariam as possíveis diferenças. Por fim, foi realizada uma análise multivariada comparando as três médias dos testes de atenção e as diferentes faixas etárias. A dispersão das pontuações nas diferentes idades pode ser visualizada na Figura 2, que mostra o desempenho no $\mathrm{AC}, \mathrm{AD}$, AA e na medida de atenção geral.

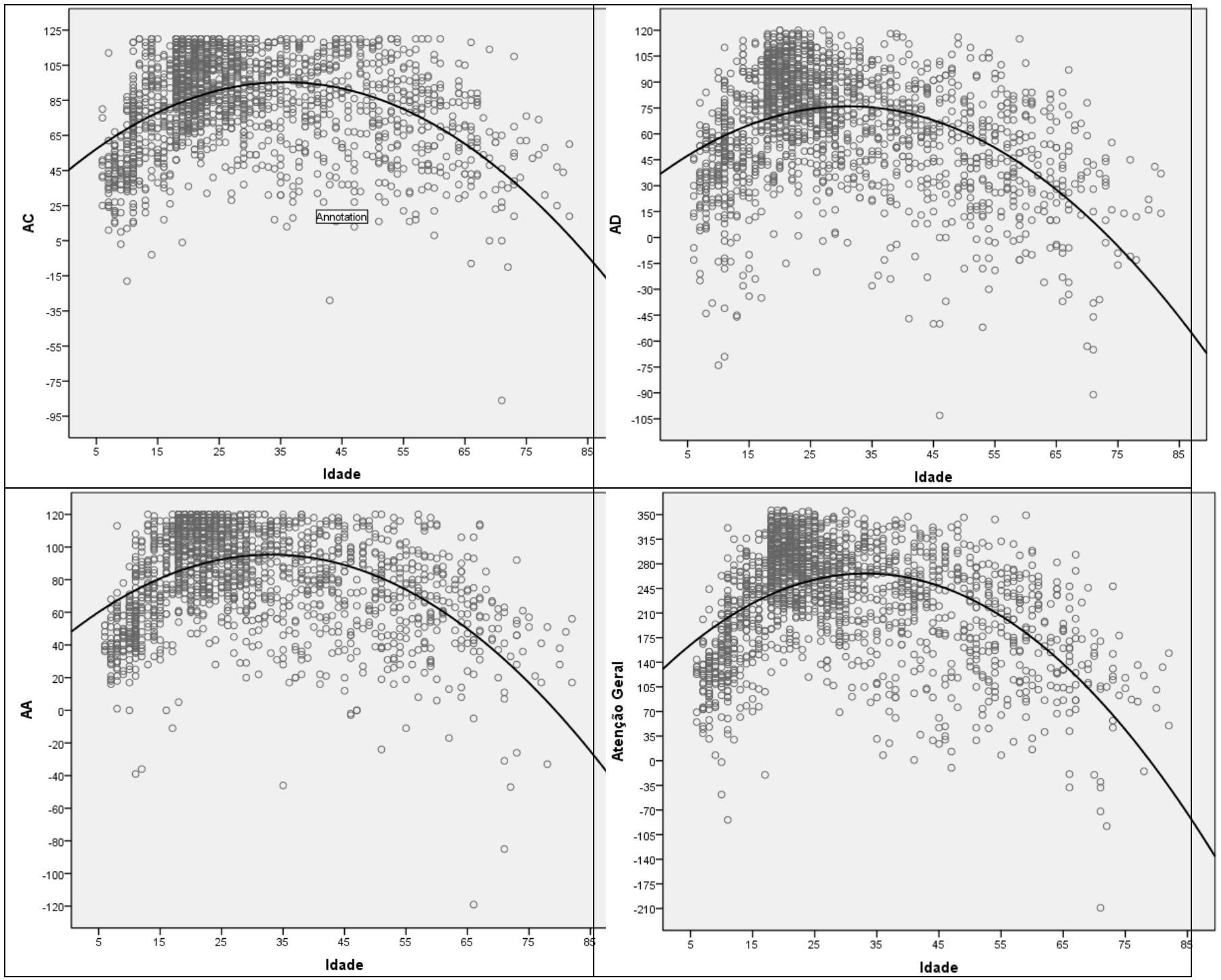

Figura 2. Gráfico de dispersão da relação entre a BPA e a idade

A Figura 2 mostra que a relação entre o construto e a idade não foi linear, mas curvilínea. Nesse sentido, o resultado está de acordo com a literatura, que indica que o nível de atenção aumenta até o final da adolescência e/ou início da idade adulta, sendo evidenciado um declínio progressivo a partir dos adultos jovens.

Para analisar essa relação, utilizou-se o modelo quadrático, observando-se que a variância explicada esteve entre 0,198, no caso do $\mathrm{AD}$, e 0,262, para a Psico-USF, Bragança Paulista, v. 18, n. 1, p. 99-108, jan./abril 2013 medida de atenção geral. A porcentagem de variância explicada é igual à correlação ao quadrado. Essa porcentagem considera o modelo quadrático, e para se obter um coeficiente de correlação calculou-se a raiz quadrada desses valores, o que mostrou valores de $r=0,45, r=0,44, r=0,47$ e $r=0,51$ para o AC, AD, AA e atenção geral respectivamente, quando correlacionados à variável idade.

Com base nessa informação, procedeu-se ao agrupamento das idades por faixas etárias, e após vários 
estudos chegou-se a uma configuração que melhor se diferenciou, não só na medida de atenção geral, mas também em cada um dos tipos de atenção avaliados. As faixas etárias formadas foram as seguintes: 6 a 10 anos, de 11 a 17 , dos 18 aos 25 , de 26 a 30 anos, pessoas de 31 a 50 anos e, por fim, indivíduos com 51 anos ou mais. O resultado da análise de variância (ANOVA) em cada tipo de atenção e na atenção geral é apresentado na Tabela 1.

Tabela 1. Valores de $F$ e $p$ nos tipos de atenção e na medida geral em função dos grupos de faixas etárias

\begin{tabular}{cccc} 
& $g l$ & $F$ & $p$ \\
\cline { 2 - 4 } AC & 5 & 133,11 & $<0,001$ \\
AD & 5 & 146,27 & $<0,001$ \\
AA & 5 & 181,83 & $<0,001$ \\
Atenção geral & 5 & 218,85 & $<0,001$ \\
\hline
\end{tabular}

A ANOVA mostrou diferença estatisticamente significativa em relação às faixas etárias formadas para cada um dos tipos de atenção e para a atenção geral, evidenciando valores de $F$ bastante elevados. Nesse sentido, a comparação das faixas etárias revelou o maior valor quando considerada a atenção geral, seguido da AA, AD e AC, respectivamente. Para verificar a pontuação de quais faixas etárias explicavam essa diferença, realizou a prova de Tukey, e os resultados podem ser observados da Tabela 2 à Tabela 5.

Tabela 2. Subconjuntos formados pela prova de Tukey em razão das faixas etárias e pontuações no AC

\begin{tabular}{cccccc}
\hline & & \multicolumn{4}{c}{ Subgrupos para alfa $=0,05$} \\
\cline { 3 - 5 } Faixas etárias & $N$ & 1 & 2 & 3 & 4 \\
\hline 6 a 10 anos & 115 & 45,22 & 68,57 & \\
51 anos ou mais & 264 & & 73,41 & & \\
11 a 17 anos & 235 & & & 86,21 & \\
31 a 50 anos & 358 & & & 91,85 & 91,85 \\
26 a 30 anos & 196 & & & 0,092 & 0,560 \\
18 a 25 anos & 591 & 1,000 & 0,215 & \\
\hline$p$ & 1.759 & & &
\end{tabular}

Tabela 3. Subconjuntos formados pela prova de Tukey em razão das faixas etárias e pontuações no AD

\begin{tabular}{|c|c|c|c|c|c|c|}
\hline \multirow[b]{2}{*}{ Faixas etárias } & \multirow[b]{2}{*}{$N$} & \multicolumn{5}{|c|}{ Subgrupos para alfa $=0,05$} \\
\hline & & 1 & 2 & 3 & 4 & 5 \\
\hline 6 a 10 anos & 115 & 35,48 & & & & \\
\hline 51 anos ou mais & 264 & 38,06 & & & & \\
\hline 11 a 17 anos & 235 & & 52,74 & & & \\
\hline 31 a 50 anos & 358 & & & 62,54 & & \\
\hline 26 a 30 anos & 196 & & & & 72,49 & \\
\hline 18 a 25 anos & 591 & & & & & 85,27 \\
\hline$p$ & 1.759 & 0,925 & 1,000 & 1,000 & 1,000 & 1,000 \\
\hline
\end{tabular}

Tabela 4. Subconjuntos formados pela prova de Tukey em razão das faixas etárias e pontuações no AA

\begin{tabular}{|c|c|c|c|c|c|c|c|}
\hline \multirow[b]{2}{*}{ Faixas etárias } & \multirow[b]{2}{*}{$N$} & \multicolumn{6}{|c|}{ Subgrupos para alfa $=0,05$} \\
\hline & & 1 & 2 & 3 & 4 & 5 & 6 \\
\hline 6 a 10 anos & 115 & 44,34 & & & & & \\
\hline 51 anos ou mais & 264 & & 60,75 & & & & \\
\hline 11 a 17 anos & 235 & & & 72,63 & & & \\
\hline 31 a 50 anos & 358 & & & & 81,99 & & \\
\hline 26 a 30 anos & 196 & & & & & 90,30 & \\
\hline 18 a 25 anos & 591 & & & & & & 102,12 \\
\hline
\end{tabular}




\begin{tabular}{llllllll}
\hline$p$ & 1.759 & 1,000 & 1,000 & 1,000 & 1,000 & 1,000 & 1,000 \\
\hline
\end{tabular}

Tabela 5. Subconjuntos formados pela prova de Tukey em razão das faixas etárias e pontuações na atenção geral

\begin{tabular}{|c|c|c|c|c|c|c|c|}
\hline \multirow[b]{2}{*}{ Faixas etárias } & \multirow[b]{2}{*}{$N$} & \multicolumn{6}{|c|}{ Subgrupos para alfa $=0,05$} \\
\hline & & 1 & 2 & 3 & 4 & 5 & 6 \\
\hline 6 a 10 anos & 115 & 125,03 & & & & & \\
\hline 51 anos ou mais & 263 & & 167,38 & & & & \\
\hline 11 a 17 anos & 235 & & & 198,79 & & & \\
\hline 31 a 50 anos & 358 & & & & 230,75 & & \\
\hline 26 a 30 anos & 196 & & & & & 254,64 & \\
\hline 18 a 25 anos & 591 & & & & & & 282,81 \\
\hline$p$ & 1.759 & 1,000 & 1,000 & 1,000 & 1,000 & 1,000 & 1,000 \\
\hline
\end{tabular}

A prova de Tukey realizada nas diferentes medidas mostrou uma diferenciação em todas as faixas etárias analisadas na medida de AA e de atenção geral. A esse respeito pode ser comentado que a menor pontuação foi apresentada pelas crianças de 6 a 10 anos, seguida das pessoas com 51 anos ou mais, ou seja, tanto as crianças como as pessoas de mais idade tendem a ter um desempenho atencional menor. Por sua vez, os indivíduos entre 18 e 25 anos apresentaram a maior pontuação média, o que condiz com a literatura (Hawkins, Kramer \& Capaldi, 1992; Papalia \& Olds, 2000), que menciona que no início da idade adulta produz-se o pico de desenvolvimento da capacidade atencional, havendo posteriormente um decréscimo gradativo.

No que se refere à medida de $\mathrm{AD}$, apenas cinco grupos de idade foram diferenciados. Contudo, as faixas etárias de 6 a 10 e de 51 anos ou mais, embora tenham sido agrupadas, continuaram sendo as que apresentaram as menores pontuações médias. Assim sendo, o resultado está de acordo com o obtido no AA e na atenção geral, no sentido de que as crianças e as pessoas mais velhas apresentam os menores desempenhos.

Por sua vez, no AC a separação de grupos não foi tão nítida, haja vista que das seis faixas etárias foram diferenciados quatro grupos. $\mathrm{Na}$ Tabela 2 pode ser observado que o menor desempenho também aconteceu nas crianças, seguido pelas pessoas de mais idade, que se agruparam com os participantes de 11 a 17 anos. Vale destacar que continuam sendo grupos extremos de idade que podem ser considerados como faixas etárias diferentes ao considerar a análise do construto da atenção. Já as pessoas de 18 a 25 anos continuaram apresentando o melhor desempenho, mesmo que a faixa etária dos 26 aos 30 anos tenha ficado alocada no mesmo agrupamento.

Resumindo, os resultados alcançados permitem inferir que a capacidade atencional dos sujeitos estudados se diferencia em razão das suas idades. Psico-USF, Bragança Paulista, v. 18, n. 1, p. 99-108, jan./ abril 2013
Nesse sentido, foram identificadas e diferenciadas seis faixas etárias, que permitem fazer uma avaliação de cada tipo de atenção e de uma medida geral considerando a idade específica do indivíduo avaliado.

Dando seguimento às análises, e considerando que o número de estímulos-alvo e distratores é o mesmo no AC, AD e AA, foi analisado o efeito da idade nos três testes. A Figura 3 mostra o efeito dessa interação.

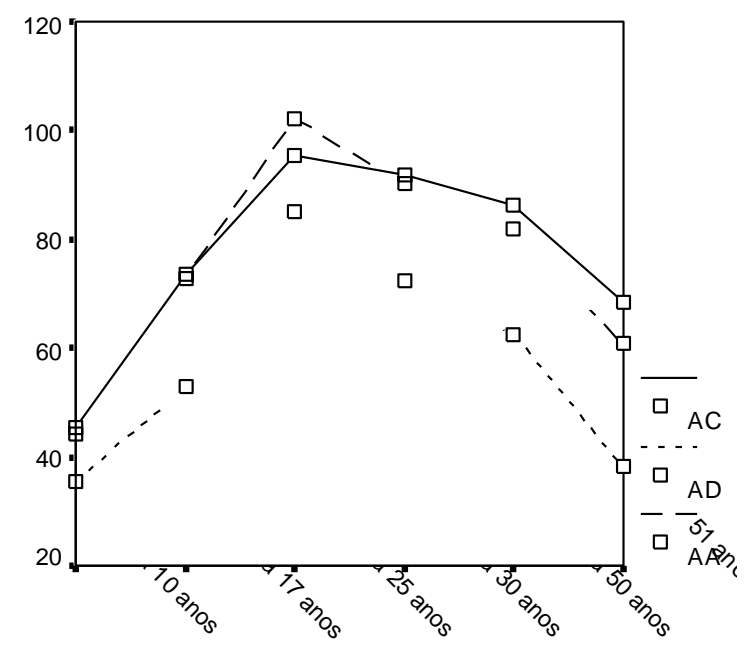

Faixas etárias

Figura 3. Gráfico da interação dos testes da BPA com as faixas etárias

Pela Figura 3, podem ser observados alguns aspectos. O primeiro mostra que o efeito da idade interage de forma diferente com o tipo de teste. Nesse sentido, evidencia-se que a atenção dividida é o tipo atencional que apresenta o menor desempenho, independentemente da faixa etária estudada. Por sua vez, ao considerar o AC e o AA, percebe-se que até o final da adolescência o desempenho de ambos testes é 
bastante semelhante, observando-se nas faixas etárias dos adultos jovens um desempenho superior em atenção alternada, enquanto a atenção concentrada apresenta um desempenho aparentemente superior a partir dos 30 anos. O segundo aspecto verificado se refere ao fato de os tipos de atenção terem apresentado diferenças estatisticamente significativas, pois ao comparar a média dos três testes foi observado um valor de $F=6,01 \quad(p=0,014)$. Ainda, também foi estatisticamente significativa a interação entre o tipo de atenção e as faixas etárias $(F=21,57, \quad p<0,001)$, indicando que o desempenho em todos os teste está relacionado com a variável idade.

\section{Discussão}

Por ser um construto complexo e com uma ampla conceituação na literatura, a atenção tem sido foco de estudo de muitos pesquisadores (Allport, 1993; Davies \& Parasuraman, 1982; Eysenck \& Keane, 2007; Pashler, 1999; Posner, 1993; Sternberg, 2008; Treisman \& Gelade, 1980; entre outros). Dentre as variáveis mais investigadas relacionadas ao desempenho de sujeitos em testes de atenção, encontra-se a idade. Conforme apontam Papalia e Olds (2000) e Sanchez-Gil e PerezMartinez (2008), existe um aumento significativo no desempenho em testes de atenção até a idade de adulto jovem, seguido de perda significativa em virtude do processo de envelhecimento. Dessa forma, o presente estudo objetivou averiguar como ocorreria o desempenho de sujeitos com diferentes idades, desde crianças até idosos, ao responderem à Bateria Psicológica para Avaliação da Atenção (BPA).

Os resultados evidenciaram, corroborando a literatura recuperada (Hawkins, Kramer \& Capaldi, 1992; Hazin \& cols., 2012; Lima, Travaini \& Ciasca, 2009; Pesce \& cols., 2005; Rueda, 2011; Rueda \& cols., 2008; Lima, Travaini \& Ciasca, 2009), que existem diferenças significativas no desempenho de testes de atenção entre diferentes faixas etárias. Essas diferenças não ocorrem da mesma forma, já que na infância, conforme o aumento da idade, existe uma melhora no desempenho (Hazin \& cols., 2012; Lima, Travaini \& Ciasca, 2009) e com o envelhecimento esse desempenho sofre uma perda significativa, ou seja, os idosos, quando comparados com grupos de adultos, apresentam um resultado inferior (Hawkins, Kramer \& Capaldi, 1992; Pesce \& cols., 2005; Rueda, 2011; Rueda \& cols., 2008). Porém, vale destacar que, como afirmam Papalia e Olds (2000), embora essas diferenças pareçam ser uma consonância na literatura, o ponto de corte para diferenciar uma faixa etária da outra não é claramente especificado. No caso da presente pesquisa, as diferenças verificadas estão de acordo com a literatura, no que se refere ao desenvolvimento da capacidade de atenção até o início da vida adulta, seguido de um leve decréscimo, que fica mais acentuado nas idades mais avançadas. Porém, as idades que compreendem cada uma das faixas etárias podem ser passíveis de alterações em outros estudos.

No que se refere à diferenciação das idades, o estudo permitiu identificar seis faixas etárias que se diferenciaram significativamente. Ao considerar os estudos recuperados, pode ser verificado que os mesmos trabalharam com dois (Hawkins, Kramer \& Capaldi, 1992) ou três grupos (Pesce \& cols., 2005) de diferentes idades ou, ainda, que as pesquisas que investigaram diferenças em relação às idades identificaram apenas três grupos que se diferenciaram nitidamente (Rueda, 2011; Rueda \& cols., 2008). Nesse sentido, vale destacar que a presente pesquisa trabalhou com idades variando de 6 até os 82 anos, o que não foi verificado em outros estudos, que pesquisaram grupos a partir dos 18 anos, bem como grupos de crianças e/ou adolescentes.

Ainda no que se refere à diferenciação em seis faixas etárias, isso mostra que a pesquisa contou com uma ampla amostra, porém, deve-se chamar a atenção para a não-diferenciação das crianças entre seis e 10 anos, e os adolescentes entre 11 e 17, uma vez que essas idades formaram apenas dois grupos. Nesse sentido, seria esperado que entre os seis e 10 anos houvesse uma maior diferenciação de grupos, assim como entre os 11 e 17, haja vista que são fases do desenvolvimento que estão em constante mudança, e nas quais os aspectos relacionados à capacidade cognitiva se desenvolvem mais rapidamente (Papalia \& Olds, 2000). Dessa forma, aventa-se a possibilidade de que a ampliação da amostra de crianças e adolescentes possa permitir a separação dos escores médios em outras faixas etárias, considerando as particularidades dessas populações. Essa mesma possiblidade pode ser pensada na faixa etária de 51 anos ou mais, pois acredita-se que o aumento da amostra de idosos permitirá uma separação maior de cada uma das idades.

No que tange ao desempenho nos três testes, o estudo mostrou que há diferenças em virtude da taxonomia, uma vez que a atenção dividida parece exigir um tipo de resposta mais complexa e que demandaria mais do respondente do que a atenção concentrada e alternada. Nesse sentido, a operacionalização do $\mathrm{AD}$ solicita que o respondente assinale como estímulo-alvo três possibilidades de resposta, enquanto que no $\mathrm{AC}$ e no $\mathrm{AA}$ o respondente deve focar sua atenção em apenas um estímulo. No caso do AC, durante dois minutos no mesmo; já no 
AA, esse estímulo muda em cada linha do teste, porém durante toda a linha ele se mantém o mesmo. Essa diferença no desempenho do teste em função do tipo de tarefa exigida merece outros estudos, pois fica evidente que os tipos de atenção e a sua operacionalização não são iguais.

\section{Considerações finais}

Considerando que o estudo proposto verificou diferenças no nível atencional em razão das diferentes faixas etárias, abarcando desde crianças até idosos, e que essa avaliação foi feita por meio de uma bateria de testes de atenção que inclui três tipos (atenção concentrada, dividida e alternada), parece importante fazer algumas considerações. Primeiro, deve-se mencionar o fato de o instrumento utilizado ser uma 'Bateria', o que se mostra como um diferencial, uma vez que no Brasil atualmente não há baterias de testes para avaliação do construto da atenção. Uma verificação no Sistema de Avaliação de Testes Psicológicos (SATEPSI) permitiu identificar 23 testes para avaliação do construto, quatro deles apresentando em seu título a denominação de bateria. São eles: as Baterias de Funções Mentais para Motorista e as Baterias Gerais de Funções Mentais. Em relação a isso, ressalta-se que embora possuam o título de baterias, na instrução dos manuais os instrumentos não necessariamente devem ser todos aplicados para posteriormente oferecer uma medida da atenção geral e dos diferentes tipos de atenção. Além disso, as amostras que serviram para construção dos instrumentos foram diferentes, o que impossibilita realizar inferências com base na resposta a todos os instrumentos.

Por fim, vale a pena destacar que, por ser uma bateria, permite uma avaliação da atenção em três diferentes formas de operacionalização, assim como uma medida geral do construto. Isso pode contribuir para uma avaliação mais completa e consistente da capacidade atencional. Também é importante destacar o fato de a BPA englobar diferentes faixas etárias, uma vez que conforme apontado na literatura, essa variável relaciona-se diretamente com o processo atencional, seja na sua evolução até a fase adulta jovem, seja no seu decréscimo na velhice. Aliado a isso, essa diferenciação por faixas etárias pode ser considerada uma evidência de validade de construto importante, pois é esperado esse aumento e posteriormente esse decréscimo no processo atencional.

Futuramente, sugere-se a realização de pesquisas com ampliação da amostra de crianças e adolescentes, assim como de pessoas com mais de 60 anos. Além disso, seriam interessantes estudos correlacionais com quadros de transtorno de déficit de atenção e hiperatividade (TDAH) em crianças e adolescentes, e quadros de demência em idosos. Esses estudos contribuiriam com mais evidências de validade para a Bateria Psicológica para Avaliação da Atenção (Rueda, 2013), no caso, evidências de validade de critério.

\section{Referências}

Allport, A. (1993). Attention and control. Em D. E. Meyer \& S. Kornblum (Orgs.). Attention and performance, XIV. Cambridge, MA: MIT Press.

Baños, R., \& Belloch, A. (1995). Psicopatología de la atención. Em A. Belloch \& E. Ibañez (Orgs.). Manual de psicopatología (Vol. 1). Valencia: Promolibro.

Dalgalarrondo, P. (2000). Psicopatologia e semiologia dos transtornos mentais. Porto Alegre: Artmed.

Davies, D. R., \& Parasuraman, R. (1982). The psychology of vigilance. London: Academic Press.

Eysenck, M. W., \& Keane, M. T. (2007). Limitações da atenção e do desempenho. Em M. W. Eysenck, \& M. T. Keane (Orgs.). Manual de psicologia cognitiva (5a ed., pp. 142-185). Porto Alegre: Artmed.

Fu, S. Fedota, J. R., Greenwood, P. M., \& Parasuraman, R. (2010). Dissociation of visual C1 and $\mathrm{P} 1$ components as a function of attentional load: an event-related potential study. Biological Psychology, 85, 171-178.

Hawkins, H. L., Kramer, A. F., \& Capaldi, D. (1992). Aging, exercise, and attention. Psychology and Aging, 7(4), 643-653.

Hazin, I., Falcão, J. T. R., Garcia, D., Gomes, E., Cortez, R., Maranhão, S., Menezes, T., \& Dias, M. G. B. B. (2012). Dados normativos do Teste de Atenção por Cancelamento (TCA) em estudantes do ensino fundamental. Psico (RS), 43(4), 428-436.

Lezak, M. D. (1995). Neuropsychological assessment. New York: Oxford University Press.

Lima, R. F., Travaini, P. P., \& Ciasca, S. M. (2009). Amostra de desempenho de estudantes do ensino fundamental em testes de atenção e funções executivas. Revista Psicopedagogia, 26(80), 188-199.

Nunes, C. H. S. S. \& Primi, R. (2010). Aspectos técnicos e conceituais da ficha de avaliação dos testes psicológicos. Em Conselho Federal de Psicologia (Org.). Avaliação psicológica: diretrizes na regulamentação da profissão (pp. 101-127). Brasília: CFP.

Papalia, D. E., \& Olds, S. W. (2000). Desenvolvimento bumano. São Paulo: Pioneira.

Pashler, H. E. (1999). The psychology of attention. 
Cambrigde, MA: MIT Press.

Pesce, C., Guidetti, L., Baldari, C., Tessitore, A., \& Capranica, L. (2005). Effects of aging on visual attentional focusing. Gerontology, 51(4), 266-276.

Posner, M. I. (1993). Attention before and during the decade of the brain. Em D. E. Meyer, \& S. Kornblum (Orgs.). Attention and performance, XIV. Cambridge, MA: Bradford.

Richards, J. E. (2005). The development of sustained attention in infants. Em Posner M. I. (Org.). Cognitive neuroscience of attention (pp. 342-56). New York: Guilford Press.

Rueda, F. J. M. (2011). Desempenho no teste de atenção dividida como resultado da idade das pessoas. Estudos de Psicologia, 28(2), 251-259.

Rueda, F. J. M. (2013). Bateria Psicológica para Avaliação da Atenção - BPA. São Paulo: Vetor.

Rueda, F. J. M., Noronha, A. A. P., Sisto, F. F., \& Bartholomeu, D. (2008). Evidência de validade de construto para o Teste de Atenção Sustentada. Psicologia: Ciência e Profissão, 28(3), 494-505.

Sanchez-Gil, I. Y., \& Perez-Martinez, V. T. (2008). El funcionamiento cognitivo en la vejez: atención y percepción en el adulto mayor. Revista Cubana de Medicina General Integrada, 24(2), 1-7.

Sternberg, R. J. (2008). Atenção e consciência. Em R. J. Sternberg (Org.). Psicologia cognitiva (4a ed., pp. 71114). Porto Alegre: Artmed.

Treisman, A. M., \& Gelade, G. (1980). A featureintegration theory of attention. Cognitive Psychology, 12, 97-136.

Wagner, C. J. P. (2003). Atenção visual em crianças e adolescentes: um estudo a partir do paradigma do tempo de reação. Dissertação de Mestrado, Universidade Federal do Rio Grande do Sul, Porto Alegre.

Warm, J. S. (1984). An introduction to vigilance. Em J. S. Warm (Ed.). Sustained attention in human performance (pp. 1-14). Chichester, UK: Wiley.

Zillmer, E. A., \& Spiers, M. V. (1998). Principles of clinical neuropsychology. Pacific Groove: Brooks/Cole.
Recebido em 05/10/2012

Reformulado em 10/03/2013

Aprovado em 01/04/2013

Sobre os autores:

Fabián Javier Marín Rueda é psicólogo, mestre e doutor em Psicologia pela Universidade São Francisco. É docente da graduação e do Programa de Pós-Graduação Stricto Sensu em Psicologia da Universidade São Francisco.

Rebecca de Magalhães Monteiro é psicóloga, mestre e doutora em Psicologia pela Universidade São Francisco. É docente da graduação da Faculdade Pitágoras de Belo Horizonte.

Contato com os autores:

E-mail: fabian.rueda@usf.edu.br 